Int. J. Curr. Res. Med. Sci. (2019). 5(3): 12-17

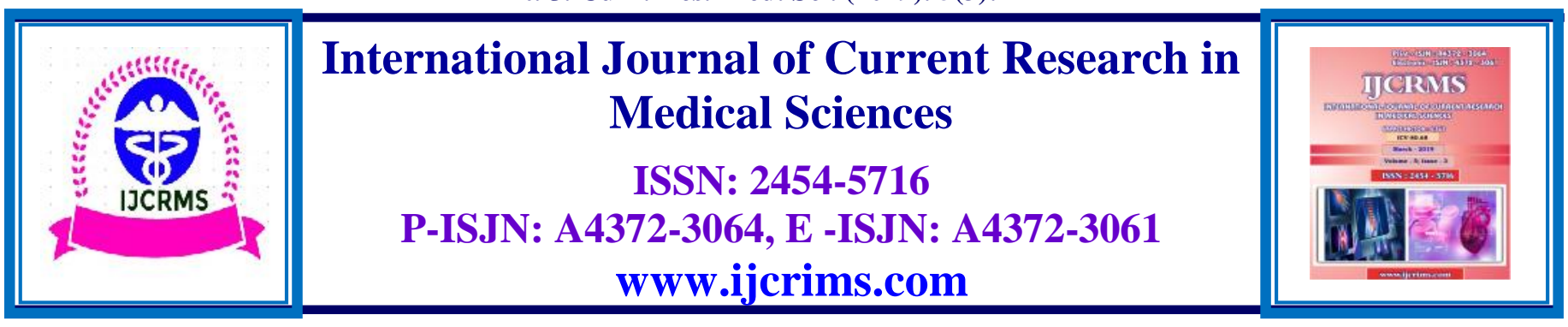

\title{
Frequency of Herpes simplex Infection in Iranian Children with Aseptic Meningitis: A systematic review and meta-analysis
}

\author{
Mohammad Hasan Mohammadi ${ }^{1}$ \\ ${ }^{1}$ Department of Pediatrics, Zabol University of Medical Sciences, Zabol, Iran
}

\section{Abstract}

Objective: the aim of this systematic review and meta-analysis was to evaluate the Frequency of Herpes Virus Infection in Iranian Children with Aseptic Meningitis.

Methods: the methods used for this systematic review were based on the "Cochrane Systematic Study Booklet" and "Appropriate Items for Systematic and Meta-Analysis Study (PRISMA)" tool.

Results: The final research was conducted on 164 participants .Based on the results of random effects model, the incidence of Herpes simplex Infection in 164 Children was 9.8\% (95\% confidence interval [CI]: 5.3, 14.3 ).

Discussion: As herpes simplex meningitis is treatable, it has to be considered for management of meningitis patients. In order to reduce the hospitalization rate and the use of unnecessary therapies and improve the health system.

Keywords: Encephalitis, herpes simplex encephalitis, Aseptic meningitis , Acyclovir, Children

\section{Introduction}

The infection of Herpes Simplex Virus is one of the most common viral infection that can affect oral mucosa, eyes, genital area, skin, brain, and ..., and create various diseases ranging from a simple infection such as recurrent herpes of the lips to the most complex and fatal kinds of infections (brain infection) (1 and 2). HSV infection can be seen in human's body in three forms i.e. initial infection, dormant form, and recurrent infection (3-5). Although HSV infection can be demonstrated in either acute or recurrent forms, it can be chronic or severe in patients having weak immune system. Initial infection is a systemic infection with various clinical manifestations (6). Initial infection depends largely on the infection area and the viral replication rate, and it occurs in patients having no prior history of infection with HSV (7).Another serious HSV infection is Herpes simplex virus (HSV) encephalitis which is one of the most common cause of acute and severe viral infection of human's central nervous system (810). Nearly $30 \%$ of HSV cases are resulted from initial infection of HSV. This infection is commonly observed in people younger than 18 (11). Almost $50 \%$ of patients with HSVare not older than 50 years old.HSV infection occurs with the prevalence rate of 1 inevery 500,000 people; the rate is rather odd (12).HSV infection results in a severe disease and death in individuals who are not treated or don't receive effective antiviral treatment for any reason(13). In patients who are not treated, a death rate of higher than $70 \%$ has been reported; only $20 \%$ of the patients have 
acquired the complete recovery of brain's normal function (14). The initial diagnosis of this infection and treatment with acyclovir are highly significant in reducing the mortality rate and the incidence of neurological complications in the survivors. Herpes Simplex Virus is also likely to create infections in face, mucosal areas, genital areas, and other skin parts (15).Another diagnostic method for HSV infection is applying polymerase chain reaction (PCR); this method is widely used in clinics and laboratories (16).From the PCR methods, Real Time TaqMan PCR has various benefits including speed, accuracy, and quantity (17). Real Time TaqMan PCR has been recently applied as a useful tool in clinical virology in diagnosing HSV genome.the aim of this systematic review and meta-analysis was to evaluate the Frequency of Herpes Virus Infection in Iranian Children with Aseptic Meningitis.

\section{Methods}

\section{Eligibility criteria}

the methods used for this systematic review were based on the "Cochrane Systematic Study Booklet" and "Appropriate Items for Systematic and Meta-Analysis Study (PRISMA)" tool. MEDLINE application was used to search other databases. In addition, PROSPERO was used to provide a systematic search that was completed recently. To search for headlines and abstracts, boolean (AND, OR, NOT), mesh, coordinate \{truncation $\}^{*}$ and related words were used; following keywords were used to provide a comprehensive context: Encephalitis, Herpes Simplex Encephalitis(HSE), Acyclovir, Child, ADEM , and prevalence rate and percent. According to the research protocol, two researchers observed the titles and abstracts separately according to the eligibility criteria; in the next step, after the removal of repeated studies, the full text of the paper was studied based on the eligibility criteria and the required information was extracted. To assess the quality of the methodology and bias risk, each observation study was evaluated using a tool developed by Hoy et al; The risk of abuse was assessed by two researchers separately and possible disparity of ideas was resolved by consensus.Meta-analysis was performed using the STAT 14 statistical software.

\section{Results}

\section{Selecting eligible papers and researches}

In the initial search on various databases, a total of 242 articles were reviewed, 211 of which turned out to be repetitive during screening process of title and abstract. 21 articles were removed due to unrelated title; out of the remaining 10 articles, 3 articles met the inclusion criteria. Of the 7 articles that were removed, 3 were reviews, 1 were letters to editors, 1 had no complete text, and 2 had low quality and could not be considered in the research. (Figure 1) 
Int. J. Curr. Res. Med. Sci. (2019). 5(3): 12-17

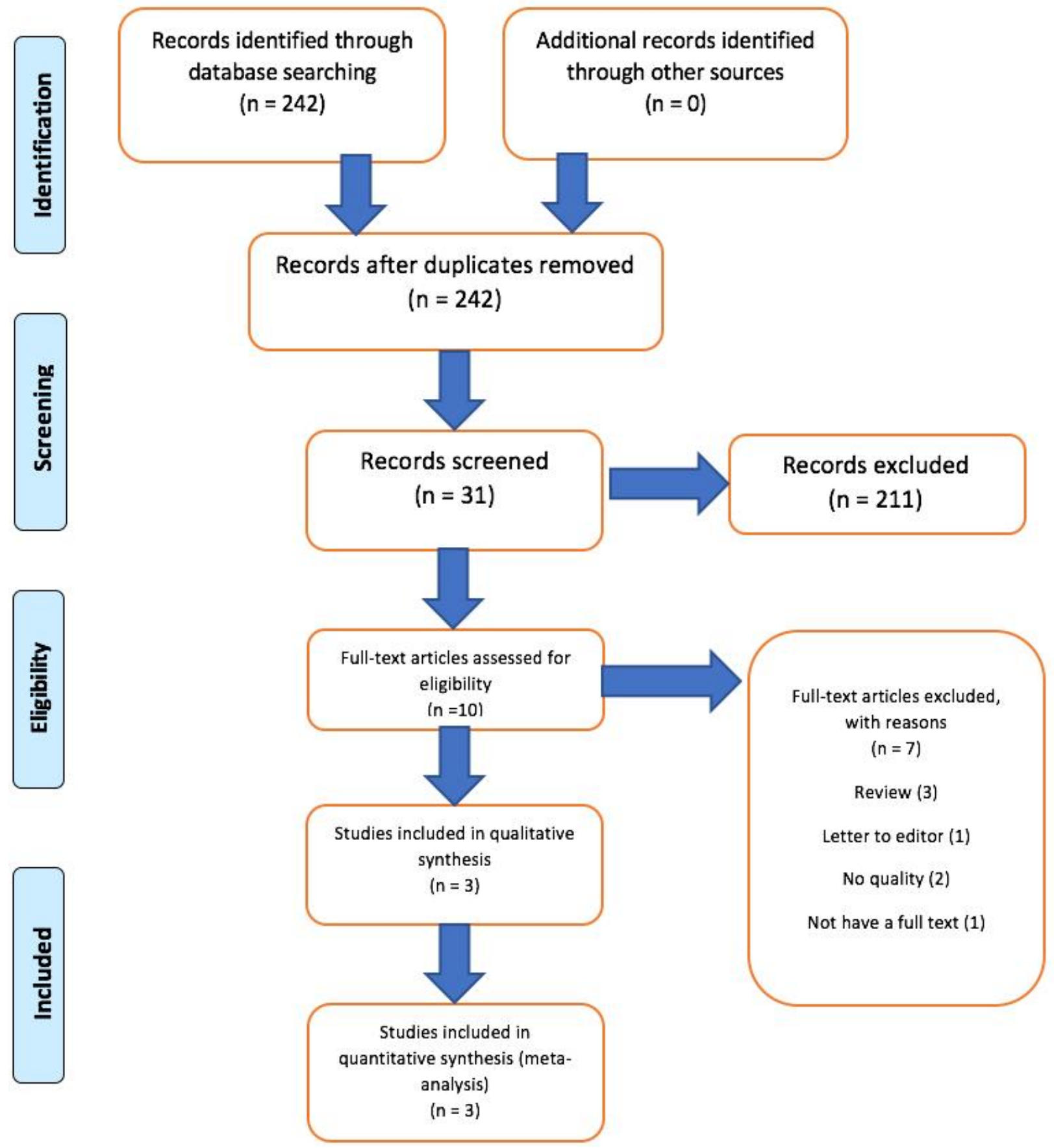

Fig 1. Prisma flow chart

\section{Characteristics of the researches and papers}

The final research was conducted on 164 participants; a cross-sectional design was used in all studies. Research was conducted in only 3 provinces out of 31 provinces of Iran.Studies, were from Bojnourd, Tehran, Mashhad; all of papers were conducted on outpatient cases $(n=3)$ . Required data was collected through interview $(\mathrm{n}=3)$ and had a low bias risk $(\mathrm{n}=3)($ Table 1$)$. 
Int. J. Curr. Res. Med. Sci. (2019). 5(3): 12-17

Table 1: Characteristics of final included studies about Frequency of Herpes simplex Infection in in Iranian Children with Aseptic Meningitis

\begin{tabular}{|c|c|c|c|c|c|}
\hline ID & Author & Year & N & frequency & Bias \\
\hline 1 & Safabakhsh & 2014 & 38 & $13.1 \%$ & Low \\
\hline 2 & Nourbakhsh & 2004 & 71 & $07 \%$ & Low \\
\hline 3 & Sasan & 2017 & 55 & $14 \%$ & Low \\
\hline
\end{tabular}

Meta-analysis Frequency of Herpes simplex Infection in Iranian Children with Aseptic Meningitis:
Children was $9.8 \%$ (95\% confidence interval [CI]: $5.3,14.3)$.

Based on the results of random effects model, the incidence of Herpes simplex Infection in 164

Table 2: Frequency of Herpes simplex Infection in in Iranian Children with Aseptic Meningitis

\begin{tabular}{|l|l|l|l|l|l|}
\hline Study & year & ES & \multicolumn{2}{l|}{$\begin{array}{l}95 \% \\
\text { Interval }\end{array}$} & conf \\
\cline { 3 - 5 } & & & low & up & \\
\hline Safabakhsh & 2014 & 0.131 & 0.025 & 0.237 & 17.80 \\
\hline Nourbakhsh & 2004 & 0.070 & 0.011 & 0.129 & 57.67 \\
\hline Sasan & 2017 & 0.140 & 0.050 & 0.230 & 24.53 \\
\hline $\begin{array}{l}\text { Overall random } \\
\text { pooled ES }\end{array}$ & ---------- & 0.098 & 0.053 & 0.143 & 100 \\
\hline
\end{tabular}

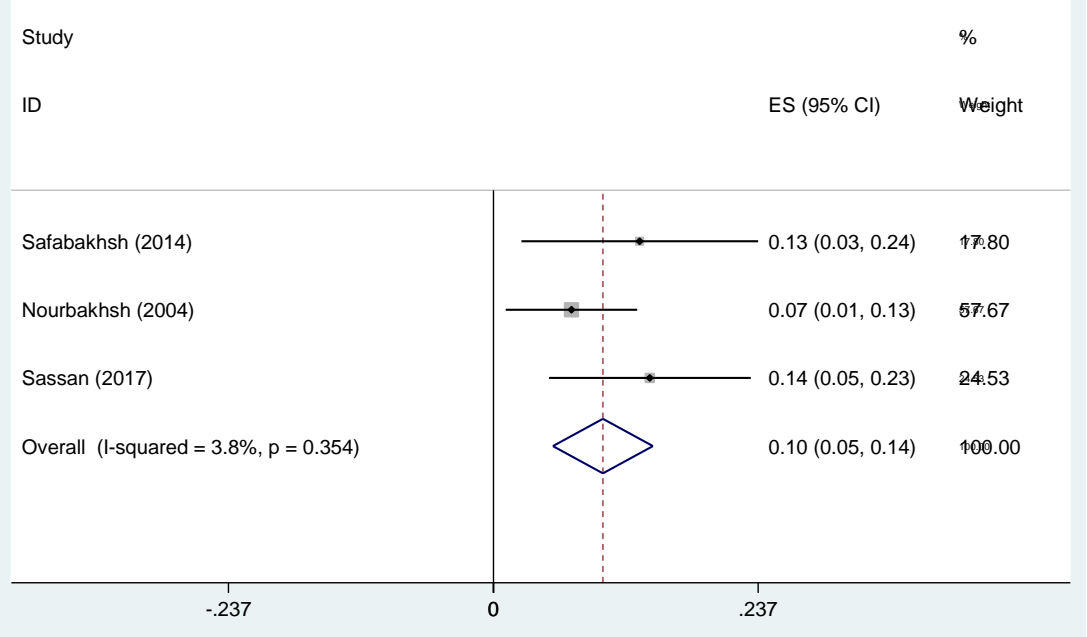

Fig.2. The Frequency of Herpes simplex Infection in Iranian Children with Aseptic Meningitis patientsand its 95\% interval for the studied cases according to the year and the city where the study was conducted based on the model of the random effects model. The midpoint of each section of the line estimates the $\%$ value and the length of the lines showing the $95 \%$ confidence interval in each study. 


\section{Discussion}

Based on the results of random effects model, the incidence of Herpes simplex Infection in 164 Children was $9.8 \%$ (95\% confidence interval [CI]: $5.3,14.3)$. The infections caused by HSVs are included as the common infections in the world. Clinically speaking, herpes viruses cause a wide variety of diseases. The common HSV infections are skin infections and mucosal infections that can result in lesions on face, mouth, genital areas, and other parts of the body (18). Moreover, this virus brings about infections and injuries in the individual's eyesas well as his/her central nervous system (CNS) (13). Herpes viruses are included as rather common viral agents in children creating systemic diseases especially nervous system involvements. High mortality of Meningoencephalitis caused by different kinds of herpes viruses and its possible complications are the main causes of quick diagnosis of the virus. The treatment of the aforementioned complications in children can prevent the disease progress and stop its progressive course (10). Encephalitis or the inflammation of the brain parenchymais a serious disease having numerous complications in children; numerous factors can create encephalitis. Herpes encephalitis is the most common cause of sporadic fatal encephalitis worldwide. Given the effectiveness of acyclovir as an antiviral drug, early diagnosis and treatment of the disease is of high significance and it is associated with a 30-to-70-percent recovery (15).HSV has been known as the most common cause of acute sporadic cases of encephalitis worldwide; HSV accounts for $10-20 \%$ of annual cases of encephalitis in the United States (17). In individuals with immunodeficiency, this infection can be highly fatal. In addition to the initial infection with this virus, the activation of the previous infection can bring about the symptoms of the disease as well.

\section{Conclusion}

As herpes simplex meningitis is treatable, it has to be considered for management of meningitis patients. In order to reduce the hospitalization rate and the use of unnecessary therapies and improve the health system.

\section{References}

1. Whitley RJ, Kimberlin DW. Herpes simplex: encephalitis children and adolescents. InSeminars in pediatric infectious diseases 2005 Jan 1 (Vol. 16, No. 1, pp. 17-23). WB Saunders.

2. Irani DN. Aseptic meningitis and viral myelitis. Neurologic clinics. 2008 Aug 1;26(3):635-55.

3. Iyer S, Mittal MK, Hodinka RL. Herpes zoster and meningitis resulting from reactivation of varicella vaccine virus in an immunocompetent child. Annals of emergency medicine. 2009 Jun 1;53(6):792-5.

4. Steiner I, Benninger F. Update on herpes virus infections of the nervous system. Current neurology and neuroscience reports. 2013 Dec 1;13(12):414.

5. Tyler KL. Herpes simplex virus infections of the central nervous system: encephalitis and meningitis, including Mollaret's. HERPESCAMBRIDGE-. 2004 Jun;11:57A-64A.

6. Mukai AO, Krebs VL, Bertoli CJ, Okay TS. TNF- $\alpha$ and IL- 6 in the diagnosis of bacterial and aseptic meningitis in children. Pediatric neurology. 2006 Jan 1;34(1):25-9.

7. Steiner I, Kennedy PG, Pachner AR. The neurotropic herpes viruses: herpes simplex and varicella-zoster. The Lancet Neurology. 2007 Nov 1;6(11):1015-28.

8. Husain EH, Mullah-Ali A, Al-Sharidah S, Azab AF, Adekile A. Infectious etiologies of transient neutropenia in previously healthy children. The Pediatric infectious disease journal. 2012 Jun 1;31(6):575-7.

9. Mehran Hesaraki, Mohammad Hasan Mohammadi. (2018). Frequency of urinary tract infection in children

10. Messacar K, Breazeale G, Robinson CC, Dominguez SR. Potential clinical impact of the film array meningitis encephalitis panel in children with suspected central nervous system infections. Diagnostic microbiology and infectious disease. 2016 Sep 1;86(1):118-20. 
11. Solomon T, Michael BD, Smith PE, Sanderson F, Davies NW, Hart IJ, Holland M, Easton A, Buckley C, Kneen R, Beeching NJ. Management of suspected viral encephalitis in adults-association of British Neurologists and British Infection Association National Guidelines. Journal of infection. 2012 Apr 1;64(4):347-73.

12. Mehran Hesaraki ,Mohammad Hasan Mohammadi. (2018). Evaluating the frequency of bacteremia in Iranian neonates admitted with jaundice: A systematic review and meta analysis. Int. J. Adv. Res. Biol. Sci. (12): 75-80

13. Messacar K, Breazeale G, Robinson CC, Dominguez SR. Potential clinical impact of the film array meningitis encephalitis panel in children with suspected central nervous system infections. Diagnostic microbiology and infectious disease. 2016 Sep 1;86(1):118-20.

14. Mohammadi MH, Hesaraki M. Acidemia in the fetus: A review. International Journal of Pharmaceutical and Biological Science Archive. 2018 May 28;6(03).

15. Zida S, Kolia-Diafouka P, Kania D, Sotto A, Foulongne V, Bolloré K, Ouangraoua S, Méda $\mathrm{N}$, Carrère-Kremer S, Van de Perre $\mathrm{P}$, Tuaillon E. Combined testing for herpes simplex virus and Mycobacterium tuberculosis DNA in cerebrospinal fluid of patients with aseptic meningitis in Burkina Faso, West Africa.
Journal of clinical laboratory analysis. 2018 Nov 25:e22719.

16. Whitley RJ. Herpes simplex virus infections of the central nervous system. Continuum: Lifelong Learning in Neurology. 2015 Dec 1;21(6, Neuroinfectious Disease):1704-13.

17. Messacar K, Breazeale G, Wei Q, Robinson CC, Dominguez SR. Epidemiology and clinical characteristics of infants with human parechovirus or human herpes virus- 6 detected in cerebrospinal fluid tested for enterovirus or herpes simplex virus. Journal of medical virology. 2015 May;87(5):829-35.

18. SASAN MS, ASHRAFZADEH F, JARAHI L, KAVYANI S. Epidemiology Of Pediatrics Encephalitis in Mashhad

19. Nourbakhsh S, Tonkaboni H, Asghaee M, Hosseini F, Vahed L, Tabatabaee A. Detection of Herpes Virus Infection Frequency in Aseptic Meningitis in Children Admitted to Rasoul-e-Akram\&Mofid Hospitals. Razi Journal of Medical Sciences. 2004 Dec 15;11(42):659-65.

20. SafamaneshS,AzimianA,Hashemi S. Survey on the prevalence of herpesvirus encephalitis in children with symptomatic aspetic meningitis in Imam Reza Hospital, Bojnourd.2014.

\begin{tabular}{|c|l|}
\hline \multicolumn{2}{|c|}{ Access this Article in Online } \\
\hline & Website: \\
\hline & www.ijcrims.com \\
\hline Quick Response Code & Subject: \\
\hline
\end{tabular}

How to cite this article:

Mohammad Hasan Mohammadi. (2019). Frequency of Herpes simplex Infection in Iranian Children with Aseptic Meningitis: A systematic review and meta-analysis. Int. J. Curr. Res. Med. Sci. 5(3): 1217.

DOI: http://dx.doi.org/10.22192/ijcrms.2019.05.03.003 